\title{
FREQUENCY ANALYSIS OF A DOUBLE-WALLED NANOTUBES SYSTEM
}

\author{
Anita Ciekot, Stanisław Kukla \\ Institute of Mathematics, Czestochowa University of Technology \\ Częstochowa, Poland \\ anita.ciekot@im.pcz.pl,stanislaw.kukla@im.pcz.pl
}

\begin{abstract}
In the present paper, a problem of free vibration of a double-walled nanotubes system is considered. The nanotubes of the system are coupled by an arbitrary number of elastic rings. The Green's functions method was used to obtain a solution to the problem. Numerical results are presented graphically.
\end{abstract}

Keywords: nanotubes system, free vibration, Green's functions

\section{Introduction}

The nanostructures, especially carbon nanotubes, have many novel electrical, chemical, mechanical and thermal properties like: almost perfect geometrical structure and extremely high strength and low mass density. These properties make the carbon nanotubes a promising technology for future applications in different fields of nanoscience and nanotechnology.

Three different theories of modelling the vibrational behavior of nanotubes: Euler-Bernoulli beam theory, the Timoshenko beam theory or the Reddy beam theory [1] have been used in the study of the properties of single-walled, double-walled and multi-walled carbon nanotubes. The study of vibration in multi-walled carbon nanotubes is a topic of papers [2, 3]. Murmu, McCarthy and Adhikari report the effect of longitudinal magnetic field on the transverse vibration of double-walled carbon nanotubes. The paper [3] by Natsuki et al. is devoted to the vibration characteristics of embedded double-walled carbon nanotubes subjected to axial pressure. The authors consider this problem by using an elastic continuum mechanics model with the van der Waals interaction between the inner and outer nanotubes.

The solution of the problems of free vibration of double-walled nanotubes can be obtained by applying methods such as those used in the classical beam theories. The explicit expressions for the natural frequencies were presented in paper [4]. The Bubnov-Galerkin and Petrov-Galerkin method were applied to derive the natural frequencies. 
In the present paper, a solution to the free vibration problem of a system of two nanotubes coupled by an arbitrary number of elastic rings is presented. The solution is obtained by using the Green's function method [5].

\section{Equations of motion of double-walled nanotubes system}

The governing equations of longitudinal vibrations of the double-walled nanotubes system are [2]:

$$
\begin{aligned}
& E_{1} I_{1} \frac{\partial^{4} w_{1}}{\partial x^{4}}-\eta A_{1} H_{x}^{2} \frac{\partial^{2} w_{1}}{\partial x^{2}}+\rho_{1} A_{1} \frac{\partial^{2} w_{1}}{\partial t^{2}}-\left(e_{0} a\right)^{2}\left(\rho_{1} A_{1} \frac{\partial^{4} w_{1}}{\partial x^{2} \partial t^{2}}-\eta A_{1} H_{x}^{2} \frac{\partial^{4} w_{1}}{\partial x^{4}}\right)= \\
& \sum_{j=1}^{n} c_{j}\left[w_{2}\left(x_{2 j}, t\right)-w_{1}\left(x_{1 j}, t\right)\right] \delta\left(x_{1}-x_{1 j}\right)+ \\
& -\left(e_{0} a\right)^{2} \sum_{j=1}^{n} c_{j}\left[\frac{\partial^{2} w_{2}}{\partial x^{2}}\left(x_{2 j}, t\right)-\frac{\partial^{2} w_{2}}{\partial x^{2}} w_{1}\left(x_{1 j}, t\right)\right] \delta\left(x_{1}-x_{1 j}\right) \\
& E_{2} I_{2} \frac{\partial^{4} w_{2}}{\partial x^{4}}-\eta A_{2} H_{x}^{2} \frac{\partial^{2} w_{2}}{\partial x^{2}}+\rho_{2} A_{2} \frac{\partial^{2} w_{2}}{\partial t^{2}}-\left(e_{0} a\right)^{2}\left(\rho_{2} A_{2} \frac{\partial^{4} w_{2}}{\partial x^{2} \partial t^{2}}-\eta A_{2} H_{x}^{2} \frac{\partial^{4} w_{2}}{\partial x^{4}}\right)= \\
& \sum_{j=1}^{n} c_{j}\left[w_{2}\left(x_{2 j}, t\right)-w_{1}\left(x_{1 j}, t\right)\right] \delta\left(x_{2}-x_{2 j}\right)+ \\
& -\left(e_{0} a\right)^{2} \sum_{j=1}^{n} c_{j}\left[\frac{\partial^{2} w_{2}}{\partial x^{2}}\left(x_{2 j}, t\right)-\frac{\partial^{2} w_{2}}{\partial x^{2}} w_{1}\left(x_{1 j}, t\right)\right] \delta\left(x_{2}-x_{2 j}\right)
\end{aligned}
$$

where $w_{i}$ is the displacement, $H_{x}$ is the strength of the longitudinal magnetic field, $\rho_{i}$ is the mass density, $E_{i}$ is the modulus of elasticity, $A_{i}$ is the area of crosssection of the $i$-th nanotubes, $\delta(\cdot)$ denotes the Dirac delta function, $x_{1}, x_{2}$ are axial positions along the nanotubes, $x_{1 j}, x_{2 j}, \mathrm{j}=1,2 \ldots n$ are points of the nanotubes which are joined by a $j$-th elastic ring, $c_{j}$ is an elastic ring constant, $e_{0}$ is a constant appropriate to nanotubes material and $a$ is an internal characteristic size. The functions $w_{i}(x, t)$ satisfy the following boundary conditions:

$$
\begin{gathered}
w_{i}(0, t)=\frac{\partial^{2} w_{i}}{\partial x_{i}^{2}}(0, t)=0, \quad i=1,2 \\
w_{i}\left(L_{i}, t\right)=\frac{\partial^{2} w_{i}}{\partial x_{i}^{2}}\left(L_{i}, t\right)=0, \quad i=1,2
\end{gathered}
$$


A sketch of the considered system of two nanotubes connected by $n$-elastic rings is shown in Figure 1.

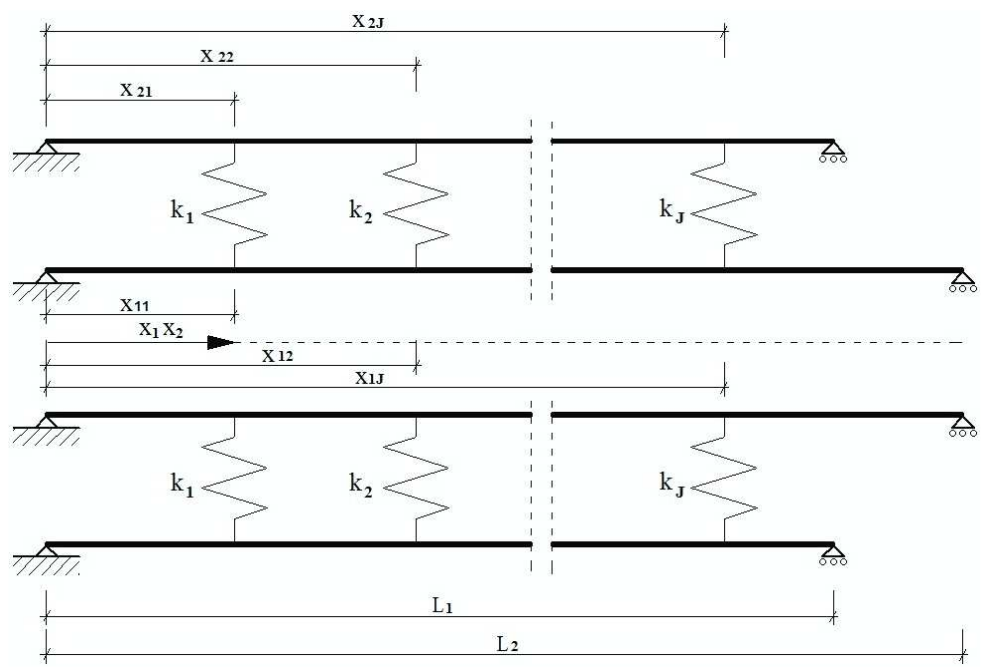

Fig. 1. Schematic diagram of the double-walled nanotubes system

\section{Solution of the problem}

The partial differential equations (1) and (2) which govern the transverse vibrations of the nanotubes can be solved assuming the solution in the form

$$
w_{i}(x, t)=\bar{W}_{i}(x) \cdot e^{i \omega t} \quad i=1,2
$$

where $\omega$ is the circular frequency. Introducing new variables: $\xi_{i}=\frac{x_{i}}{L}, W_{i}=\frac{\bar{W}_{i}}{L_{i}}$ into equations (1)-(2), the following non-dimensional equations can be obtained:

$$
\begin{aligned}
& \frac{d^{4} W_{1}}{d \xi_{1}^{4}}-F_{1} \frac{d^{2} W_{1}}{d \xi_{1}^{2}}-\Omega^{4} P W_{1}\left(\xi_{1}\right)= \\
& P \frac{1}{h} \sum_{j=1}^{n} \gamma_{j}\left[W_{2}\left(\xi_{2 j}\right)-h W_{1}\left(\xi_{1 j}\right)\right] \delta\left(\xi_{1}-\xi_{1 j}\right)+ \\
& -P \mu^{2} \sum_{j=1}^{n} \gamma_{j}\left[h \frac{d^{2} W_{2}}{d \xi_{2}^{2}}\left(\xi_{2 j}\right)-\frac{d^{2} W_{1}}{d \xi_{1}^{2}}\left(\xi_{1 j}\right)\right] \delta\left(\xi_{1}-\xi_{1 j}\right)
\end{aligned}
$$




$$
\begin{aligned}
& \frac{d^{4} W_{2}}{d \xi_{2}^{4}}-F_{2} \frac{d^{2} W_{2}}{d \xi_{2}^{2}}-\frac{r s \Omega^{4}}{h^{4}} Q W_{2}\left(\xi_{2}\right)= \\
& \frac{1}{h^{3}} s Q \sum_{j=1}^{n} \gamma_{j}\left[W_{2}\left(\xi_{2 j}\right)-h W_{1}\left(\xi_{1 j}\right)\right] \delta\left(\xi_{2}-\xi_{2 j}\right)+ \\
& -\frac{\mu^{2} s}{h} Q \sum_{j=1}^{n} \gamma_{j}\left[\frac{d^{2} W_{2}}{d \xi_{2}^{2}}\left(\xi_{2 j}\right)-\frac{1}{h} \frac{d^{2} W_{1}}{d \xi_{1}^{2}}\left(\xi_{1 j}\right)\right] \delta\left(\xi_{2}-\xi_{2 j}\right)
\end{aligned}
$$

where: $\Omega^{4}=\frac{\rho_{1} A_{1} L_{1}^{4}}{E_{1} I_{1}} \omega^{2}, \gamma_{j}=\frac{c_{j} L_{1}^{3}}{E_{1} I_{1}}, \mu=\frac{e_{0} a}{L_{1}}, s=\frac{E_{1} I_{1}}{E_{2} I_{2}}, r=\frac{\rho_{2} A_{2}}{\rho_{1} A_{1}}, \quad q=\frac{\eta A_{1} H_{x}^{2} L_{1}^{2}}{E_{1} I_{1}}$, $h=\frac{L_{1}}{L_{2}}, \quad p=\frac{A_{2}}{A_{1}}, \quad F_{1}=\left(q-\Omega^{4} \mu^{2}\right) P \quad P=\frac{1}{\left(1+\mu^{2} q\right)}, \quad F_{2}=\frac{s\left(p q-\mu^{2} \Omega^{4} r\right)}{h^{2}}$, $Q=\frac{1}{\left(1+\mu^{2} p q s\right)}$.

Taking into account equation (5) in equations (3)-(4) the following boundary conditions for the function $W_{i}(\xi)$ can be obtained

$$
\begin{gathered}
W_{i}(0)=W_{i}^{\prime \prime}(0)=0, \quad i=1,2 \\
W_{i}\left(L_{i}\right)=W_{i}^{\prime \prime}\left(L_{i}\right)=0, \quad i=1,2
\end{gathered}
$$

The solution of the problem (6)-(9) can be presented in the form [5]:

$$
\begin{gathered}
W_{1}\left(\xi_{1}\right)=\frac{P}{h} \sum_{j=1}^{n} \gamma_{j}\left[W_{2}\left(\xi_{2 j}\right)-h W_{1}\left(\xi_{1 j}\right)\right] G_{1}\left(\xi_{1}, \xi_{1 j}\right)+ \\
-P \mu^{2} h \sum_{j=1}^{n} \gamma_{j}\left[\frac{d^{2} W_{2}}{d \xi_{2}^{2}}\left(\xi_{2 j}\right)-\frac{1}{h} \frac{d^{2} W_{1}}{d \xi_{1}^{2}}\left(\xi_{1 j}\right)\right] G_{1}\left(\xi_{1}, \xi_{1 j}\right) \\
W_{2}\left(\xi_{2}\right)=\frac{1}{h^{3}} s Q \sum_{j=1}^{n} \gamma_{j}\left[W_{2}\left(\xi_{2 j}\right)-h W_{1}\left(\xi_{1 j}\right)\right] G_{2}\left(\xi_{2}, \xi_{2 j}\right)+ \\
-\frac{\mu^{2} s}{h} Q \sum_{j=1}^{n} \gamma_{j}\left[\frac{d^{2} W_{2}}{d \xi_{2}^{2}}\left(\xi_{2 j}\right)-\frac{1}{h} \frac{d^{2} W_{1}}{d \xi_{1}^{2}}\left(\xi_{1 j}\right)\right] G_{2}\left(\xi_{2}, \xi_{2 j}\right)
\end{gathered}
$$

where $G_{i}\left(\xi_{i}, \eta_{i}\right)$ are the Green's functions. These functions satisfy the differential equations 


$$
\begin{gathered}
\frac{\partial^{4} G_{1}}{\partial \xi_{1}^{4}}+F_{1} \frac{\partial^{2} G_{1}}{\partial \xi_{1}^{2}}-P \Omega^{4} G_{1}\left(\xi_{1}\right)=\delta\left(\xi_{1}-\eta_{1}\right) \\
\frac{\partial^{4} G_{2}}{\partial \xi_{2}^{4}}+F_{2} \frac{\partial^{2} G_{2}}{\partial \xi_{2}^{2}}-\frac{r s \Omega^{4} Q}{h^{4}} G_{2}\left(\xi_{2}\right)=\delta\left(\xi_{2}-\eta_{2}\right)
\end{gathered}
$$

and the boundary conditions analogous to the conditions (8)-(9):

$$
\begin{gathered}
G_{i}\left(0, \eta_{i}\right)=\left.\frac{\partial^{2} G_{i}}{\partial \xi_{i}^{2}}\right|_{\xi_{i}=0}=0 \\
G_{i}\left(L_{i}, \eta_{i}\right)=\left.\frac{\partial^{2} G_{i}}{\partial \xi_{i}^{2}}\right|_{\xi_{i}=L_{i}}=0
\end{gathered}
$$

The derivation of the Green's functions has been presented in paper [6].

The equations (10)-(11) are satisfied for all values of independent variables $\xi_{1}, \xi_{2}$, particularly for $\xi_{1}=\xi_{1 i}, \xi_{2}=\xi_{2 i},(i=1,2, \ldots n)$ :

$$
\begin{gathered}
W_{1}\left(\xi_{1 i}\right)=\frac{P}{h} \sum_{j=1}^{n} \gamma_{j}\left[W_{2}\left(\xi_{2 j}\right)-h W_{1}\left(\xi_{1 j}\right)\right] G_{1}\left(\xi_{1 i}, \xi_{1 j}\right)+ \\
-\mu^{2} P h \sum_{j=1}^{n} \gamma_{j}\left[\frac{d^{2} W_{2}}{d \xi_{2}^{2}}\left(\xi_{2 j}\right)-\frac{1}{h} \frac{d^{2} W_{1}}{d \xi_{1}^{2}}\left(\xi_{1 j}\right)\right] G_{1}\left(\xi_{1 i}, \xi_{1 j}\right) \\
W_{2}\left(\xi_{2 i}\right)=\frac{s Q}{h^{3}} \sum_{j=1}^{n} \gamma_{j}\left[W_{2}\left(\xi_{2 j}\right)-h W_{1}\left(\xi_{1 j}\right)\right] G_{2}\left(\xi_{2 i}, \xi_{2 j}\right)+ \\
-\frac{\mu^{2} s Q}{h} \sum_{j=1}^{n} \gamma_{j}\left[\frac{d^{2} W_{2}}{d \xi_{2}^{2}}\left(\xi_{2 j}\right)-\frac{1}{h} \frac{d^{2} W_{1}}{d \xi_{1}^{2}}\left(\xi_{1 j}\right)\right] G_{2}\left(\xi_{2 i}, \xi_{2 j}\right)
\end{gathered}
$$

Similarly, the second order derivative of the functions $W_{1}\left(\xi_{1 i}\right)$ and $W_{2}\left(\xi_{2 i}\right)$ determined by using equations (10)-(11) satisfy the equations:

$$
\begin{aligned}
& \frac{\partial^{2} W_{1}\left(\xi_{1 i}\right)}{\partial \xi_{1}^{2}}=\frac{P}{h} \sum_{j=1}^{n} \gamma_{j}\left[W_{2}\left(\xi_{2 j}\right)-h W_{1}\left(\xi_{1 j}\right)\right] \frac{\partial^{2} G_{1}}{\partial \xi_{1}^{2}}\left(\xi_{1 i}, \xi_{1 j}\right)+ \\
& -\mu^{2} h P \sum_{j=1}^{n} \gamma_{j}\left[\frac{d^{2} W_{2}}{d \xi_{2}^{2}}\left(\xi_{2 j}\right)-\frac{1}{h} \frac{d^{2} W_{1}}{d \xi_{1}^{2}}\left(\xi_{1 j}\right)\right] \frac{\partial^{2} G_{1}}{\partial \xi_{1}^{2}}\left(\xi_{1 i}, \xi_{1 j}\right)
\end{aligned}
$$




$$
\begin{aligned}
& \frac{\partial^{2} W_{2}\left(\xi_{2 i}\right)}{\partial \xi_{2}^{2}}=\frac{1}{h^{3}} s Q \sum_{j=1}^{n} \gamma_{j}\left[W_{2}\left(\xi_{2 j}\right)-h W_{1}\left(\xi_{1 j}\right)\right] \frac{\partial^{2} G_{2}}{\partial \xi_{2}^{2}}\left(\xi_{2 i}, \xi_{2 j}\right)+ \\
& -\frac{\mu^{2} s}{h} Q \sum_{j=1}^{n} \gamma_{j}\left[\frac{d^{2} W_{2}}{d \xi_{2}^{2}}\left(\xi_{2 j}\right)-\frac{1}{h} \frac{d^{2} W_{1}}{d \xi_{1}^{2}}\left(\xi_{1 j}\right)\right] \frac{\partial^{2} G_{2}}{\partial \xi_{2}^{2}}\left(\xi_{2 i}, \xi_{2 j}\right)
\end{aligned}
$$

Introducing a new constant

$$
V_{\mathrm{i}}=W_{2}\left(\xi_{2 i}\right)-h W_{1}\left(\xi_{1 i}\right)-\mu^{2} h^{2}\left(\frac{\partial^{2} W_{2}\left(\xi_{2 i}\right)}{\partial \xi_{2}^{2}}-\frac{1}{h} \frac{\partial^{2} W_{1}\left(\xi_{1 i}\right)}{\partial \xi_{1}^{2}}\right)
$$

into equations (16)-(19), after some transformations we obtain the system of equations which can be written in the form:

$$
\sum_{j=1}^{n}\left(\gamma_{j} A_{i j}-\delta_{i j}\right) V_{j}=0, \quad i=1,2 \ldots . n
$$

where

$$
A_{i j}=\left(\frac{s Q}{h^{3}} G_{2}\left(\xi_{2 i}, \xi_{2 j}\right)-P G_{1}\left(\xi_{1 i}, \xi_{1 j}\right)\right)-\mu^{2}\left(\frac{s Q}{h} \frac{\partial^{2} G_{2}}{\partial \xi_{2}^{2}}\left(\xi_{2 i}, \xi_{2 j}\right)-P \frac{\partial^{2} G_{1}}{\partial \xi_{1}^{2}}\left(\xi_{1 i}, \xi_{1 j}\right)\right)
$$

This system of equations has a solution if and only if it satisfies the condition

$$
\operatorname{det}\left[\gamma_{j} A_{i j}-\delta_{i j}\right]=0
$$

This equation is solved numerically with respect to natural frequencies of the $\Omega$.

\section{Numerical results}

The system of double-walled carbon nanotubes with identical physical properties and the same length was analyzed. The nanotubes of the system are connected by two elastic rings at $\xi_{11}=0.3$ and $\xi_{12}=0.7$ of the first nanotube and $\xi_{21}=0.3$ and $\xi_{22}=0.7$ of the second nanotube. The strength of the longitudinal magnetic field $H_{x}=0$, was assumed.

The computations for four different values of the nondimensional rings constants: $c_{1}=c_{1}=1 ; 10 ; 20 ; 50$, have been performed. The four dimensionless natural vibration frequencies as functions of parameter $\mu$ were calculated for this system and these are plotted in Figure 2. The Maple package [7] was used for calculations.

The curves in the Figure 2 showed that for the first two eigenfrequencies $\Omega_{1}, \Omega_{2}$, the values of the rings constants are particularly relevant. Moreover, it is 
observed that an increase of the parameter characterized the nanotubes (material and internal characteristic size) causes a decrease of the frequencies for all considered values of the rings constants.
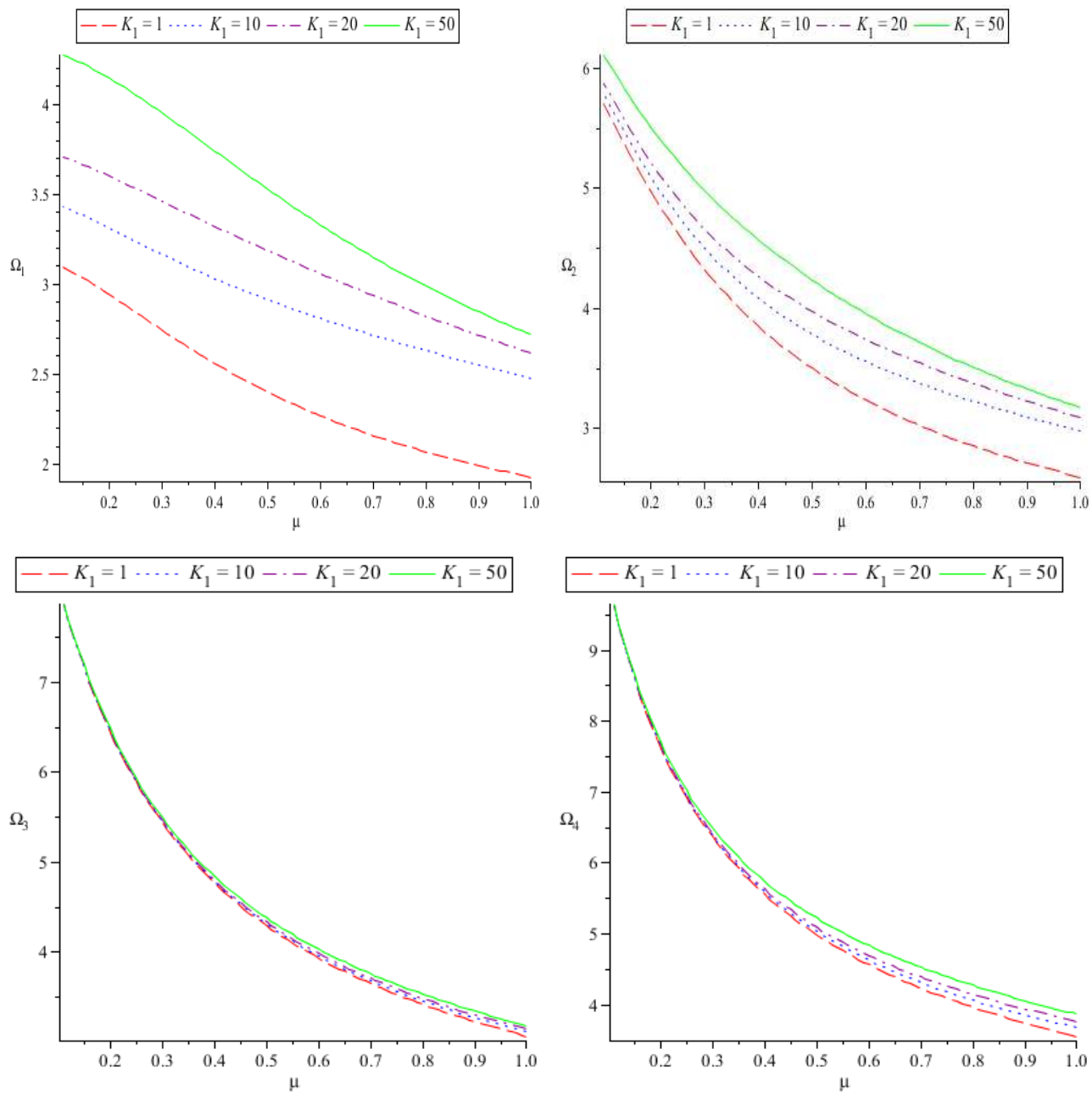

Fig. 2. The first four dimensionless natural vibration frequencies as a function of $\mu$

\section{Conclusions}

In this paper the Green's function method was applied to solve the problem of free vibration of double-walled nanotubes coupled by $n$-elastic rings. Simplysupported boundary conditions were employed in this study. It is observed that as the parameter $\mu$ increases, the frequencies of the nanotubes decrease. In the presented example the number of nanotubes and coupling rings was limited to two, 
but the approach can be used to solve the problems of systems consisting of many nanotubes coupled by elastic rings.

\section{References}

[1] Ansari R., Sahmani S., Small scale effect on vibrational response of single-walled carbon nanotubes with different boundary conditions based on nonlocal beam models, Commun. Nonlinear Sci. Numer. Simulat. 2012, 17, 1965-1979.

[2] Murmu T., McCarthy M.A., Adhikari S., Vibration response of double-walled carbon nanotubes subjected to an externally applied longitudinal magnetic field: a nonlocal elasticity approach, Journal of Sound and Vibration 2012, 331, 5069-5086.

[3] Natsuki T., Lei X. W., Shi J.X., Ni Q.Q., Vibration characteristics of embedded double-walled carbon nanotubes subjected to an axial pressure, 18 International Conference on Composite Materials.

[4] Elishakoff I., Pentaras D., Fundamental natural frequencies of double-walled carbon nanotubes, Journal of Sound and Vibration 2009, 322, 652-664.

[5] Duffy D.G., Green's Functions with Applications, Chapman \& Hall/CRC, 2001.

[6] Ciekot A., Kukla S., Frequency analysis of a double-nanobeam-system, Journal of Applied Mathematics and Computational Mechanics 2014, 1(13), 23-31.

[7] Richards D., Advanced Mathematical methods with Maple, Cambridge University Press, 2009. 